\title{
Springs, clutches and motors: driving forward kinetochore mechanism by modelling
}

\author{
Elina Vladimirou • Ed Harry • Nigel Burroughs • \\ Andrew D. McAinsh
}

Published online: 18 February 2011

(C) The Author(s) 2011. This article is published with open access at Springerlink.com

\begin{abstract}
As a mechanical system, the kinetochore can be viewed as a set of interacting springs, clutches and motors; the problem of kinetochore mechanism is now one of understanding how these functional modules assemble, disassemble and interact with one another to give rise to the emergent properties of the system. The sheer complexity of the kinetochore system points to a future requirement for data-driven mathematical modelling and statistical analysis based on quantitative empirical measurement of sister kinetochore trajectories. Here, we review existing models of chromosome motion in the context of recent advances in our understanding of kinetochore molecular biology.

Keywords chromosome directional instability . kinetochores $\cdot$ mathematical modelling $\cdot$ microtubule . mitosis
\end{abstract}

Responsible Editors: James Wakefield and Herbert Macgregor

E. Vladimirou $\cdot$ E. Harry $\cdot$ A. D. McAinsh $(\bowtie)$

Centre for Mechanochemical Cell Biology, Warwick

Medical School, University of Warwick,

Coventry, UK

e-mail: andrew@mechanochemistry.org

E. Harry

Molecular Organisation and Assembly in Cells (MOAC)

Doctoral Training Centre, University of Warwick,

Coventry, UK

E. Harry $\cdot$ N. Burroughs

Warwick Systems Biology Centre, University of Warwick, Coventry, UK

$\begin{array}{ll}\text { Abbreviations } & \\ \text { AP movement } & \text { Anti-poleward movement } \\ \text { ATP } & \text { Adenosine triphosphate } \\ \text { DIC } & \text { Differential interference contrast } \\ \text { GTP } & \text { Guanosine triphosphate } \\ \text { J\&H } & \text { Joglekar and Hunt } \\ \text { k-fibre } & \text { Kinetochore fibre } \\ \text { kMT } & \text { Kinetochore microtubule } \\ \text { MTs } & \text { Microtubules } \\ \text { P movement } & \text { Poleward movement } \\ \text { PEF } & \text { Polar ejection force } \\ \text { 3D } & \text { Three dimensional }\end{array}$

\section{Introduction}

Mitosis, the set of processes by which sister chromatid pairs are first captured by spindle microtubules, shuttled into a central position (in metaphase) and subsequently segregated to opposite poles of the cell (in anaphase), is fundamental to all eukaryotic life. This complex series of movements is to a large extent driven by kinetochores, which are adaptive, multilayered mechanochemical machines that assemble at the centromere of each sister chromatid and engage the plus ends of k-fibres, bundles of around 20-25 kinetochore microtubules (kMTs) that emanate from the spindle poles (for review see Cleveland et al. 2003; McAinsh et al. 2003; Santaguida and Musacchio 2009). The connections between k-fibres and kineto- 
chores are remarkable in that they allow kinetochores to grip and to exert force on k-fibres during both the growth and shrinkage phases of the k-fibre (Joglekar et al. 2010). Incisive experiments using video-enhanced DIC microscopy made over 17 years ago revealed that once sister kinetochores become attached to MTs emanating from opposite poles (biorientation), they undergo a series of oscillations (Hughes and Swann 1948; Lewis 1939) that involve periods of poleward (P) and anti-poleward (AP) movement at constant velocity-a process termed chromosome directional instability (Skibbens et al. 1993). Recently, single-particle tracking algorithms and fluorescent protein reporters have been deployed to systematically track the $3 \mathrm{D}$ position of multiple sister kinetochore pairs over time in living human cells (Jaqaman et al. 2010) revealing semi-regular oscillations indicative of stochasticity in the switching mechanisms (Fig. 1a, c). During oscillations, the sister pair also move towards and away from one another (breathing), and the kinetochore itself undergoes internal deformation between the inner and outer plates (stretching; Maresca and Salmon 2009; Uchida et al. 2009). These oscillations, breathing and internal stretching reflect the adaptive switching of the kinetochores between two different structural and functional states: one that is bound to growing MTs undergoing AP movement, i.e. the trailing sister and one that binds depolymerising MTs undergoing $\mathrm{P}$ movement, i.e. the leading sister.

The chromosome-kinetochore-kMT assembly can be viewed as a set of interacting springs, clutches and motors. The physical linkage between the sister kinetochores via centromeric chromatin is generally regarded to be a flexible elastic connection (Nicklas 1988). Consistently, the cross correlation of the two biorientated sisters as they oscillate back and forth during metaphase shows fairly tight, but not complete, motion coupling (Jaqaman et al. 2010). There is, however, evidence that the centromeric linkage is plastic, although this may depend on the attachment state of the kinetochores (i.e. syntelic; Loncarek et al. 2007). In addition the, inherent multi-layered nature of the kinetochore and the observed conformation changes within the structure (Wan et al. 2009) could be thought of as constituting a second flexible elastic connection. In effect, the kinetochore-kMT attachment functions as a dynamic mechanical connection or "molecular clutch" mediated by proteins that can form physical contacts with the microtubule lattice. An attractive hypothesis is that kinetochores translate the forces generated by GTP hydrolysis-dependent growth and shrinkage of kMTs into chromosome movements, essentially rectifying MT dynamic instability into useful work. The regularity of oscillations indicates that kinetochores control force generation through regulation of $\mathrm{kMT}$ dynamics. Additional forces are generated by kinetochore-bound plus-end and minus-end translocating ATP-dependent molecular motor proteins directly exerting force on kMTs (Sharp et al. 2000) while external forces within the mitotic spindle also influence kinetochore (chromosome) motion. Most notably, the polar ejection force (PEF) pushes chromosome arms away from the spindle pole whilst depolymerisation of MTs at their minus-ends (spindle pole) generates poleward MT flux, which exerts a poleward force on the chromosome. The PEF is generated by chromosome-bound chromokinesin motors or by polymerizing MTs striking the chromosome arms (Kapoor and Compton 2002). Changes in spindle pole position and the forces exerted by the spindle as a whole also need to be considered (for review see Dumont and Mitchison 2009). Given the level of mechanical complexity in the system and the high degree of coupling between the different components, we will need mathematical models to identify concepts, determine dependencies, integrate multiple datasets consisting of empirical observations and generate novel and testable hypotheses. Here, we set out to review existing models of chromosome directional instability in mammalian cells, relate these models to recent experimental advances and discuss where future efforts are needed.

\section{Force and feedback}

There have been a number of mathematically based studies that demonstrate kinetochore oscillations, which can arise from a number of mechanisms; the key requirements are a spatially dependent force and a feedback process. In essence, as kinetochores move away from the metaphase plate, a restoring force is needed. This is provided by the PEF that increases in strength near the pole, thus increasing the antipoleward force. However, without a feedback 
a

TRAJECTORY OF SINGLE SISTER PAIR

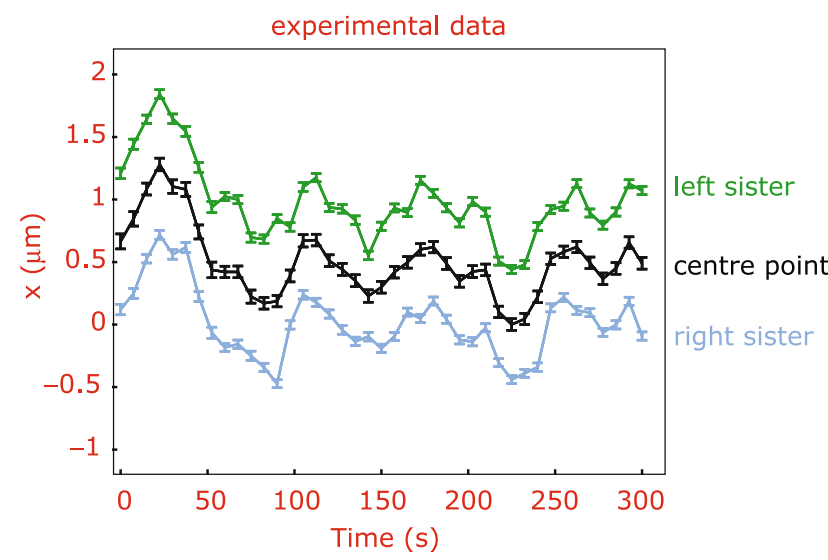

b

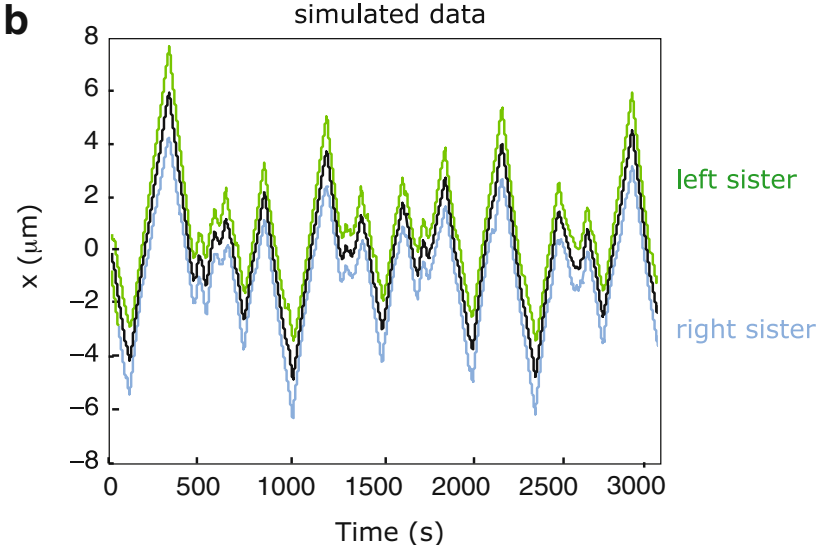

C

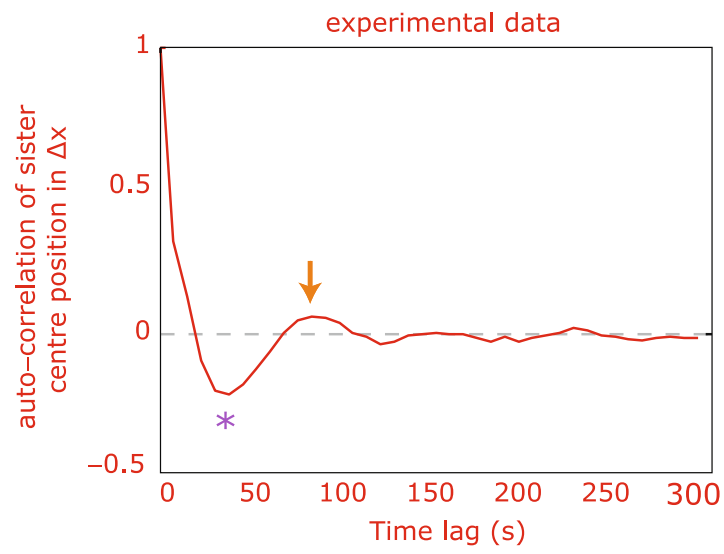

d

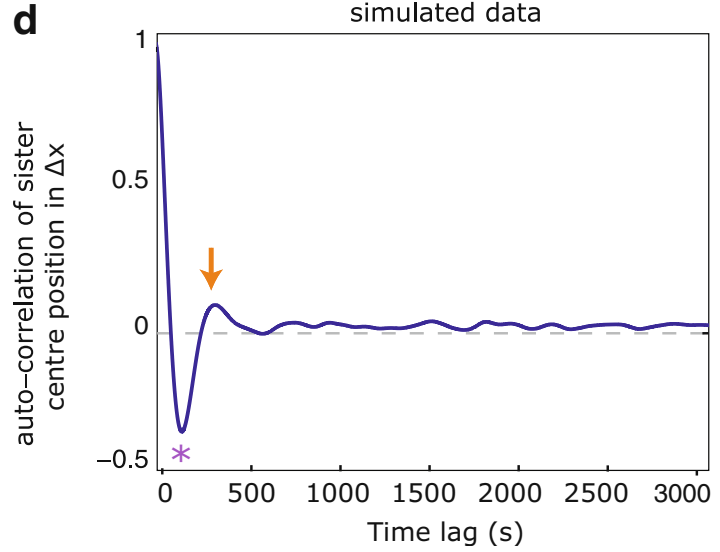

Fig. 1 Comparison of experimental and simulated data of sister kinetochore oscillations in metaphase. a Trajectory of a single sister kinetochore pair undergoing oscillations along the normal $(x)$ to the metaphase plate. Coordinate positions of right (blue line) and left (green line) sisters plus the centre point between them (black line) are from experimental data of kinetochores in human HeLa cells (Vladimirou and McAinsh, unpublished data). b Trajectory of a single sister kinetochore pair undergoing oscillations in metaphase. Data was simulated using the mathematical model from Joglekar and Hunt 2002, which is largely based on parameters from PTK-1 cells, hence the longer timescale for oscillations compared to human cells. c, d Autocorrelation of the centre positions of sister pairs from experimental (50 trajectories; red line; c) and simulated data (100 trajectories; blue line; d). The half period (purple star) and full period (orange arrow) of the oscillation in the autocorrelation curves are indicated mechanism, this would position the chromosomes stably at the metaphase plate. The models vary fundamentally in whether they incorporate MT dynamic instability, i.e. as a two-state growth/ catastrophe model or assume full regulation of MT growth processes by the kinetochore. Most of these models use force balancing either in a set of differential equations or in a mesoscopic simulation model. At the subcellular level viscous forces dominate and inertial effects are negligible, thus all the forces acting on the object can be equated to the viscous drag term that is proportional to velocity. This can be particularly useful as it can be used to calculate the system's position using the condition that the forces must balance. Force balance models have been used extensively in a biological context. For example, a force balance model was developed to explain force generation by polymerising actin filaments (Mogilner and Oster 2003). Also, the steady state pole separation during the early stages of spindle morphogenesis in Drosophila embryos was modelled by a forcebalance differential equation complemented by equations describing the distribution of MTs, motors and 
forces (Cytrynbaum et al. 2003). The assumptions and principles needed to develop such a forcebalance model are summarised in box 1 and are described further in (Howard 2005).

Box 1: Modelling chromosome directional instability using a force-balance approach

\begin{abstract}
Step 1: Assumption of overdamped dynamics: The equation of motion is given by $m d^{2} x / d t^{2}+y$ $\mathrm{dx} / \mathrm{dt}+\mathrm{kx}=\mathrm{F}$ where the first (inertial) term is Newton's second law (the acceleration of a mass is proportional to the force applied to it), the second term is due to viscous forces ( $\gamma$ is the drag coefficient and is dependent on the size and the shape of the object as well as the fluid's viscosity) and the third term is due to elastic forces (Hooke's law, the force an object will experience when connected to a spring of stiffness $k$ extending a distance $x$ beyond its resting length $x_{0}$ ). $F$ can be any other externally applied force. The above equation can describe a system that can display monotonic or oscillatory motion depending on the strength of the damping. When a microscopic system is strongly damped, the inertial term can be ignored and the equation of motion reduces into $\mathrm{y} d x / d t+k x=F$.
\end{abstract}

Step 2: Identifying the antagonistic forces acting on the system: All the forces acting on the object are either spring-like ( $k x)$ depending on position or of another functional form ( $F$ ) that may depend on position and/or time. For example the force due to an elastic attachment between one kinetochore and another would depend on the position of the kinetochore relative to the other ( $k x$ form). An example of an external force might be due to a molecular motor and could have a stochastic form depending on time. In any case, the net force is opposed by an equal drag force.

Step 3: Solving the equality to get the system's position and velocity in time as a response to the changing forces: In a complex, multicomponent system such as the kinetochorekMT mechanical unit, predicting the response of the system to the applied forces is not straight forward. By using the assumption that $\mathrm{F}-\mathrm{kx}-\mathrm{y} \mathrm{dx} / \mathrm{dt}=0$ at each point in time and space, this differential equation can be solved to determine the velocity $\mathrm{dx} / \mathrm{dt}$ and the position $x$ satisfying the force-balance.

\section{Kinetochore-microtubule attachment complex}

For mammalian systems the model of Joglekar and Hunt 2002 - from here on called J\&H-best reflects our current understanding of how kinetochores work (Gardner and Odde 2006). The heart of the model is a simple force-balance equation between the forces exerted on kinetochores by attached microtubules and the PEFs acting on the chromosome arms (Fig. 2a). This model is built on the work of Terrell Hill, who developed a beautiful model that describes how a kinetochore may follow a depolymerising MT, with the kinetochore being theorised as a sleeve containing binding sites distributed at equal distances (Hill 1985). The length of a sleeve is assumed to be $40 \mathrm{~nm}$, which is the thickness of the outer plate of a kinetochore based on electron micrographs (Brinkley et al. 1992). Since each tubulin heterodimer is $8 \mathrm{~nm}$ long, five dimers can penetrate the sleeve lengthwise, and since a microtubule is made up of 13 protofilaments circularly arranged, $5 \times 13=65$ tubulin dimers can enter a sleeve. There are, therefore, an estimated 65 binding sites per sleeve. As a MT enters a sleeve, it forms more and more bonds lowering the total free energy, so MT movement will be biased to entering the sleeve (Fig. 2b). However, moving further into the sleeve requires breaking all the previously formed bonds, so there is an ever increasing energy barrier for the MT to overcome. A microtubule can move relative to a sleeve either by depolymerising or by the sleeve moving by (forced) diffusion. In the J\&H model, the kinetochore sleeves are connected to the chromosome through springs that generate a restoring potential to reposition the sleeve near the chromosome. The MT plus-end position in a sleeve is, therefore, affected by the load on the chromosome and the polymerisation state of the kMT, with a high pulling load tending to move the MT further out of the sleeve (Fig. 2c). The overall result is that a sleeve will shift its position on a MT to balance its free energy against its restoring load. This energy balance endows the sleeve with a crucial property; sleeves tend to follow a depolymerising MT at a constant rate until the restoring load causes the sleeve to stall and the MT quickly detaches.

Experimental evidence for such a sleeve structure is growing: The core microtubule binding site in the vertebrate kinetochore is composed of the KNL-1 protein and two 4-subunit protein complexes- $\mathrm{Ndc} 80$ and Mis12/MIND - which through multiple proteinprotein interactions constitute the $\mathrm{KMN}$ network (Cheeseman et al. 2006). The Ndc80 complex can directly bind MTs with low affinity $(\sim 3 \mu \mathrm{M}$; Wei et al. 2006; Cheeseman et al. 2006). KNL-1 has also been shown to bind to MTs but with very low affinity (Cheeseman et al. 2006), thereby necessitating further 
work to define its direct involvement in kinetochorekMT attachment. Nevertheless, reconstitution of the full KMN network does lead to a synergistic increase in MT binding affinity to $\sim 0.5 \mu \mathrm{M}$ (Cheeseman et al. 2006). Recent structural studies now show that the Ndc80 complex can bind to both inter- and intratubulin dimer interfaces (Alushin et al. 2010). The Ndc80 complex can also self-assemble forming oligomeric arrays via inter-complex interactions suggesting that it could mediate multiple kinetochoreMT interactions (Alushin et al. 2010). Consistently, Salmon and colleagues demonstrated that in vivo there are at least ten copies of Ndc80 complex per microtubule in vertebrates (Johnston et al. 2010; Fig. 2b). Additional binding sites could be provided by the Ska complex, which can oligomerise and form ring-like structures on MTs in vitro with a binding affinity of $\sim 0.3 \mu \mathrm{M}$ (Welburn et al. 2009). Together, these could provide the tens of binding sites that would be organised into a sleeve-like structure as proposed by Hill. Importantly, both the Ska and Ndc80 complexes have both been shown, in optical trapping experiments, to couple a plastic bead to a depolymerising MT, with the Ndc80 complex being able to form load-bearing attachments (Welburn et al. 2009; Powers et al. 2009). At the same time, there are a multitude of molecular motors, MT-associated proteins and plus-end tracking proteins that could contribute to the interaction surfaces within the kinetochore (for review see Maiato et al. 2004). Thus, molecular evidence supports the idea that a kinetochore sleeve that incorporates multiple low-affinity binding sites (see model in Fig. 2b) can operate as a "molecular clutch" to couple MTs to the chromosome. However, modelling this complex structure and specifically its kinetics and characteristics (elasticity, energy barriers, inter-sleeve connectivity, effects on kMT (de)polymerisation and dynamic instability, length constraints) has proceeded little since the original formulation.

To account for polymerisation of kMTs, J\&H modified the Hill sleeve model as follows: During catastrophe, microtubules shrink much faster than kinetochores move, therefore only growing microtubules enter empty sleeves. Once inside a sleeve, the polymerising microtubule will switch to a depolymerising state (catastrophe) with a rate $k_{\text {cat }}$. During an oscillation, the leading sister will follow depolymerising kMTs poleward causing greater and greater load on the associated sleeves on the leading sister. The sleeves on the trailing sister attached to polymerising MTs are under low load as they are being led by the chromosome and any depolymerising kMTs are quickly lost out of the sleeves. In this configuration, the leading sister is 'pulling' the chromosome. This is supported by laser surgery experiments in which cutting kMTs of the trailing sister resulted in no change in the poleward velocity; however, cutting the kMTs of the leading sister resulted in the complete abolition of poleward movement (Khodjakov and Rieder 1996). We should also bear in mind that under certain situations the kinetochores can generate pushing forces through poleward microtubule flux (Toso et al. 2009) and that measurement of centromere deformation suggests that AP movement of a kinetochore can produce a pushing force (Skibbens et al. 1993). Eventually, the load on the leading sister's sleeves becomes too great and the depolymerising MTs detach from their sleeves. The other sister that has accumulated depolymerising MTs is now dominant and the chromosome starts to follow the new leading sister. This mechanism is consistent with exciting recent experiments on purified kinetochore particles showing that a threshold force $(\sim 4 \mathrm{pN})$ causes a decrease in the kinetochore particle-kMT attachment lifetime (Akiyoshi et al. 2010). Importantly, this work also shows that increasing the load up to this threshold actually stabilises attachments, a result that is consistent with experiments in living cells (Nicklas 1988). This mechanism is something that can be incorporated into modelling efforts (see discussion below).

The spatial gradient in the PEF is vital to this directional switch, providing the restoring force towards the spindle equator against which the leading sister competes. Since the PEF is proportional to the density of microtubules from the spindle, effectively a point source, it is reasonable to assume the force follows an inverse square distribution (PEF proportional to $1 / x^{2}$ where $x$ is the distance from the poles), although other functional forms have been used suggesting that oscillatory behaviour is robust to its exact form. The role of the PEF in chromosomal directional instability has been tested in newt lung cells by severing the chromosome arms using femtosecond-pulsed lasers (Ke et al. 2009). While the main characteristics of directional instability, such as constant speed remained unaltered, shortening the 


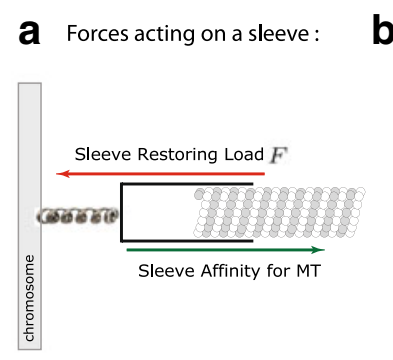

b Molecular-sleeve:

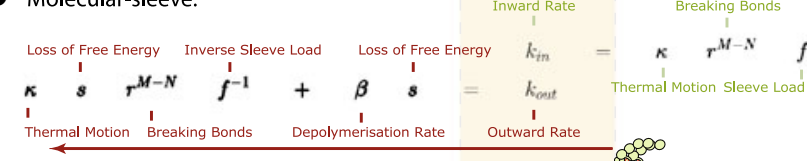

C At steady-state $k_{\text {in }}=k_{\text {out }}$ the most probable position for the MT tip is given by: $\quad N_{\max }=M+(\operatorname{lnr})^{-1} \ln \left(\frac{\kappa f\left(1-s f^{-2}\right)}{\beta s}\right)$

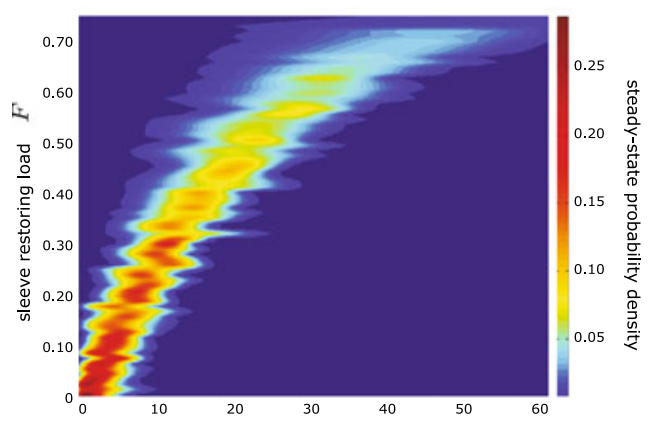

In

Out

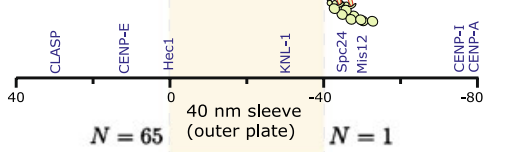

$90^{\circ}$

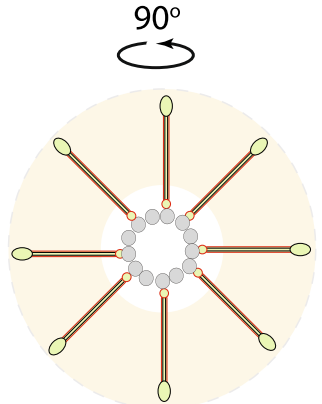

Parameter definitions:

$M=66$

$r=\mathrm{e}^{\frac{-b \leftarrow}{k_{B} T}}$ free energy of bond breaking

$s=\mathrm{e}^{\frac{a}{k_{B} T}}$

$f=\mathrm{e}^{\frac{-F l}{2 k_{B} T}}$

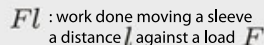

Molecular components

red outline = MT binding domains

1) $\alpha / \beta$-tubulin heterodimer: $\bigcirc$

2) Ska complex:

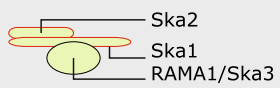

3) Mis12/MIND complex:

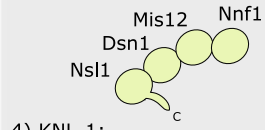

4) KNL-1:

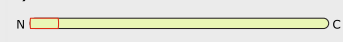

5) Ndc80 complex:

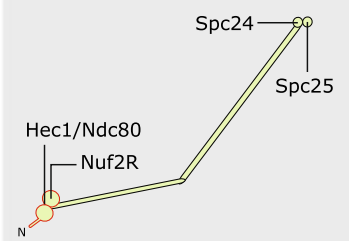

d Joglekar and Hunt model:

net polar ejection force

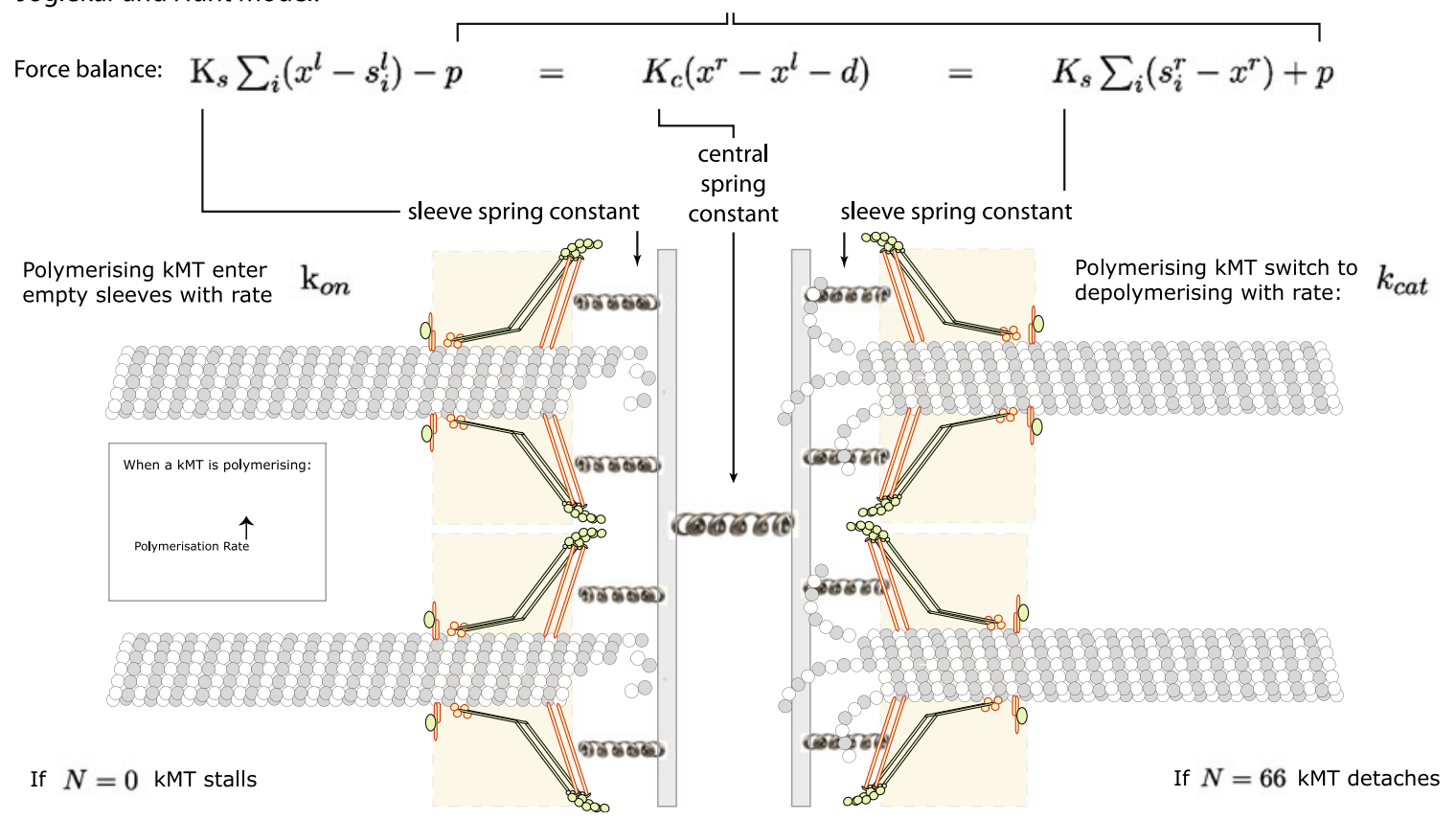


Fig. 2 Hill sleeve and Joglekar and Hunt model. a A Hill sleeve balances its affinity for a MT (green arrow) against the restoring load (red arrow) pulling it back to the chromosome. b Contemporary view (upper panel from the side; lower panel is a $90^{\circ}$ rotation showing end-on view) of a Hill sleeve. Binding sites inside the sleeve are shown as KMN networks and Ska complexes. These form multiple attachment sites for the MT as it penetrates into the sleeve, which is the outer plate of the kinetochore. The inward (green arrow) and outward (red arrow) movement rates of the MT are shown and depend on the position of the MT inside the sleeve and the restoring load on the sleeve. Position of kinetochore components within the kinetochore is based on super-resolution imaging and adapted from Wan et al. 2009. Subunit composition and organisation of complexes is based on Cheeseman et al. 2006; Maskell et al. 2010; Petrovic et al. 2010 and Wan et al. 2009. c A Monte Carlo simulation of the kMT tip position inside the sleeve gives the probability density distribution over position and load (based on rate equations from Joglekar and Hunt 2002). Higher loads on the sleeve increase the probability of MT tip shifting to positions further and further out of the sleeve. $\mathbf{d}$ The Joglekar and Hunt model is based on a force balance between the kinetochores, sleeves and polar ejection forces. The positions of the sleeves are determined by the kinetics of the sleeve-bound MT as determined by Hill's rate equations (Hill 1985). Polymerising MTs enter empty sleeves with a rate $k_{\text {on }}$ and switch to depolymerising with a rate $k_{\text {cat }} . N$ is the position of the MT tip inside the sleeve. When $N=1$ the MT is fully inserted, whereas the MT detaches from the sleeve when $N=66$

chromosome arms increased the average amplitude of oscillations indicating that the rate of directional reversals depends on the magnitude of PEF, specifically the load on the kinetochores, which is to be expected. The results lend support to the hypothesis that PEF is dependent on both the size of chromosome arms and microtubule density, and that kinetochores are responsive to external spindle forces, whilst providing experimental support for the $J \& H$ model. Ke et al. estimated the PEF distribution to increase sublinearly with the displacement $y$ from the spindle equator $\left(y^{0.57}\right)$ suggesting that the PEF increases rapidly near the equator and flattens toward the poles These experimental data differ from the previously proposed inverse square law, and it remains to be tested how incorporation of such a PEF may affect the result of the simulation compared to experiment. Such a force profile could drive oscillations around the metaphase plate, but it is harder to see how this would provide a restoring force for a mono-orientated chromosome close to one spindle pole. Nevertheless, simulation of the J\&H model using parameters estimated from Ptk1 and Newt lung cells is in good qualitative agreement with experimental data, reproducing key aspects of chromosome directional switching (Fig. 1b). Using an autocorrelation analysis (see box 2), these simulated oscillations are semi-regular with a half period of around $150 \mathrm{~s}$ (Fig. 1d) — a value that closely matches experimental measurements (Khodjakov and Rieder 1996). Importantly, the amplitude of the half and full period in the autocorrelation curve is very similar to that observed for sister kinetochore oscillations in human cells (Jaqaman et al. 2010 and compare with Fig. 1c). Thus, the J\&H model is able to generate the key characteristics of this high-level emergent behaviour.

Box 2: Assessing oscillatory behaviour using the autocorrelation function

The autocorrelation function is commonly used to identify periodicity in a sequence of events by comparing the sequence to a time-lag version of itself (lag k). The lag k autocorrelation of discretely sampled data can be estimated by:

$$
R(k)=\frac{1}{\sigma^{2}(n-k)} \sum_{t=1}^{n-k}\left(x_{t}-\mu\right)\left(x_{t+k}-\mu\right)
$$

where $x_{t}$ is the value of observation at time $t, \mu$ is the mean and $\sigma^{2}$ the variance of the sample of observations. For example, a random process exhibits no periodic behaviour giving an autocorrelation that is zero up to stochastic noise.
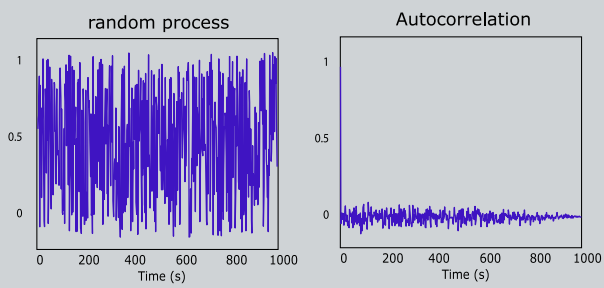

In contrast, the autocorrelation of a periodic signal, such as a sinusoidal wave, results in a plot where the first negative trough and the first positive peak represent the half and full period of the oscillation.
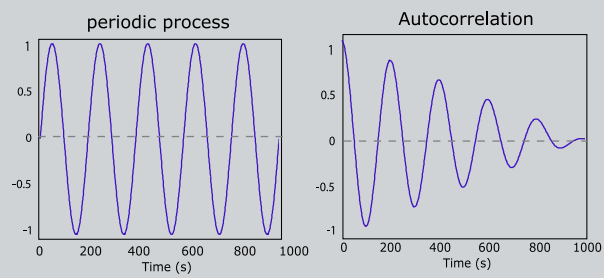

Autocorrelation is therefore a powerful statistical tool for analyzing and comparing oscillatory behaviors in biological systems (e.g. kinetochore trajectories). 
This agreement with experiment does not, however, mean that we now have a concrete mechanistic understanding of chromosomal directional instability. There are many missing behaviours and mechanisms that need to be incorporated. First, sister kinetochore pairs move in three dimensions (which can generate torque; Jaqaman et al. 2010), become stretched through forces generated by kMT dynamics (Maresca and Salmon 2009; Uchida et al. 2009) and are liable to effects from neighbouring chromosome (arms). Second, sister kinetochores undergo breathing that has a distinct period. Third, the catastrophe rate for kMTs is almost tenfold lower than that of non-kMTs in the spindle (Zhai et al. 1995) meaning that kinetochores must contain systems that slow kMT turnover. Indeed, inhibition of the aurora-B protein kinase causes a further reduction in the turnover rate (Cimini et al. 2006), whereas depletion of the CENPA NAC/CAD kinetochore complex results in a kMT turnover rate indistinguishable from non-kMTs (Amaro et al. 2010). Moreover, the CENP-H and -I subunits of this complex are asymmetrically localised to the trailing (AP moving) kinetochore, and their depletion causes more frequent directional switches and irregular oscillations (Amaro et al. 2010). The integration of such a control system will be an important next step in developing models of chromosomal directional instability. Finally, the force generating effects of kinetochore-bound molecular motors and additional external spindle forces, such as poleward MT flux, need to be considered.

A model that incorporates molecular motors and includes the effects of poleward MT flux has been described (Civelekoglu-Scholey et al. 2006). At its conception, this model had the benefit of being composed of well-characterised components, and thus the model was more constrained than earlier models. The Civelekoglu-Scholey et al. model employs a force balance approach similar to the J\&H model that is based on parameters and spindle organisation in Drosophila syncytial blastoderm embryos. It includes additional force terms, and separately considers force-balance for the kinetochores and for the kMT itself. For the case of the kinetochore, the forcebalance equation includes terms for the viscous drag forces, the net force exerted by the antagonistic action of plus-end-directed (CENP-E) and minus-enddirected (dynein) motors stepping on the kMT lattice, the force of $\mathrm{kMT}$ polymerisation pushing on the kinetochore inner plate, the PEF (modelled as a quadratic function centred at the mitotic equator) and the inter-kinetochore tension (as in $\mathrm{J} \& \mathrm{H}$ ). The presence of both minus- and plus-end-directed motors at a kinetochore generates a linkage to the kMT, one that has multiple binding sites and thus is similar to the Hill sleeve, although expends ATP when there is relative kMT motor movement. The wellcharacterised motor properties, i.e. load-velocity relationships, stall force and the maximum motor velocity essentially parametrise this 'sleeve' leaving only the number of motors per unit kMT length as free parameters. The above equation for the kinetochore is coupled to a force-balance equation for the kMT; this equation takes into account the net force generated at the plus end of the kMT engaged in the kinetochore i.e., the pulling force minus the pushing force and a balancing force due to poleward flux that is due to depolymerisation of minus-ends (at spindle poles) by depolymerisation motors. At the same time, kMT dynamic instability, i.e. stochastic switching between polymerising and depolymerising states, is incorporated. Importantly, the model includes a tension feedback in which the rates of rescue and catastrophe are modified by controlling the activity of the kinesin-13 depolymerase motor (KLP59C) through the tension acting on the kMT. This mechanism was successfully incorporated into the model of Odde and colleagues to explain chromosome congression in budding yeast (Gardner et al. 2005). Thus, when the kinetochore is under low tension, the catastrophe rate is high, driving poleward movement; when tension increases (due to PEF, cohesin stretching) then the depolymerase cannot act on the plus-end promoting switching to the polymerising state. In this way, kinetochores would "keep hold" of kMTs when under load. This aspect is consistent with the in vitro and in vivo work (described above) showing that kinetochore attachment improves with load (Akiyoshi et al. 2010; Nicklas 1988). At the mechanistic level, experimental work does support the idea that tension can regulate the activity of kinetochore components (for review see Lampson and Cheeseman 2010). However, to date, experiments show that tension may lead to the activation, rather than inactivation, of human kinesin-13 (MCAK). More work is needed to define these regulatory loops and relate the activity of MCAK and the related Kif2b to load variation. Kinesin- 8 motors may also play a key role although it 
is controversial as to whether human Kif18a is an MT depolymerase (Du et al. 2010; Mayr et al. 2007).

The above equations are solved numerically for varying numbers of kMT attachment sites. The model is able to reproduce both metaphase and anaphase (by simulating loss of cohesin) chromosome behaviour in Drosophila embryonic spindles, in which metaphase oscillations are barely detectable. The model can generate metaphase oscillations when the poleward flux rate is low and the kMT turnover rate is high enough (e.g. in budding yeast). The model cannot, however, account for oscillations in mammalian cells where the kMT turnover rate is slow. Moreover, the high dependence and role in the model on the CENP$\mathrm{E}$, dynein and MCAK molecular motors raises additional questions about the generality of the model. In human cells, depletion of CENP-E or MCAK has no effect on directional switching during metaphase (Jaqaman et al. 2010). In fact, data from human cells, instead, indicates that the depolymerising motors (MCAK and Kif18a) are required to set the speed of kinetochore movement (Jaqaman et al. 2010; Stumpff et al. 2008; Wordeman et al. 2007). Clearly, motors and sleeves must function together, and we need models to integrate these mechanisms of force generation with experimental data. Despite the modelling differences between J\&H and CivelekogluScholey et al., the kinetochore-kMT attachment models behave similarly, e.g. both give a load independent velocity suggesting that the fine detail is not crucial to emergent behaviour such as metaphase oscillations. Together with experimental work, these models support the idea that there are clearly different solutions to the "problem" of controlling chromosome motion in mitosis. Whilst the underlying mechanisms and components will be conserved, the functional dependency on them can (and does) vary between species. Both Civelekoglu-Scholey et al. and J\&H provide important starting points for future efforts.

\section{Mechanochemical feedbacks}

The last 5 years has seen limited advancement of these models, although there have been new efforts incorporating additional local mechanochemical feedbacks at the kinetochore (Liu et al. 2008; Shtylla and Keener 2010). These models are motivated by experi- ments that propose that kinetochores contain force sensitive components that are also involved in regulating kMT dynamics (Andrews et al. 2004). Liu et al. totally neglects the mechanical forces acting on the kinetochore and focuses solely on local chemical reactions within the kinetochore that respond, via a tension-sensitive protein, to control kMT dynamics; chromosome velocity is assumed linear in the activity of the tension sensor. However, the resulting oscillations around the metaphase plate are highly periodic and do not closely reflect experimental data. Shtylla and Keener used a similar tensionsensitive feedback, but integrated this with a mechanical force-balance model along the lines of J\&H. The idea is that such regulatory feedbacks will be necessary to generate more robust oscillations and transitions between attachment states (see below). Interestingly, Civelekoglu-Scholey et al. have already included a tension-sensitive feedback to drive directional switches (Civelekoglu-Scholey et al. 2006). Overall, all these models need to be more closely linked to experiment, i.e. we do not know yet whether such negative feedback loops exist to control directional switching and the role of tension as a signal. Whether kinetochores can detect changes in tension is a matter of current debate (for review see Khodjakov and Pines 2010). However, this discussion is focused on whether kMT attachment, rather than tension, is sensed by the spindle checkpoint machinery. The in vitro work by Biggins and colleagues with purified kinetochore particles clearly shows that mechanical behaviour alters under varying load (Akiyoshi et al. 2010; see earlier discussion). Here, kinetochores are only bound to a single MT so these changes must be attributed to tension changes and not attachment. Understanding how such a tensionometer operates within the kinetochore is of major importance.

\section{Sisters are not always biorientated}

The models discussed so far have all focused on the biorientated (also known as amphitelic) state and the emergence of oscillatory behaviours. Of course a kinetochore begins life unattached after nuclear envelope breakdown and can adopt different attachment states as time progresses: (1) lateral bindingkinetochore attached to the lattice of an MT, (2) monotelic - one sister bound to one pole, the other 
sister unattached, (3) syntelic - both sisters bound to one pole, (4) amphitelic-each sister bound to opposite poles and (5) merotelic-one (or two) sister (s) bound to both poles. Through a poorly understood set of processes, sister kinetochores move between these attachment states, ending up in the (stabilised) biorientated state and finding their way to the spindle equator - a process termed congression (for review see Kops et al. 2010). We, therefore, need to understand these different attachment states in terms of their behaviour and mechanism. The holy grail is to develop and run simulations of the entire process from the initial unattached state to segregation of sisters in anaphase. Models do exist for a single mono-orientated kinetochore pair although these are in effect simplifications of the biorientated state (Campas and Sens 2006; Joglekar and Hunt 2002; Liu et al. 2007). No models exist for the syntelic or lateral state. It is worth noting that the monoorientated chromosomes observed in live cell movies could be either monotelic or syntelic and that neither current data nor models distinguish between these two states. We clearly need more quantitative data and markers for these different attachment states in order to define their behaviour. A recent model by Tournier and colleagues (Courtheoux et al. 2009) made a first effort to model merotelic attachment, albeit during anaphase in fission yeast. This is a simplification of the Civelekoglu-Scholey et al. model and, as before, force-balance equations are established to describe the time evolution of the kinetochore position. The inter-chromatid tension is replaced by an outer-inner kinetochore plate tension to model merotely for a single kinetochore, whilst the chromosome drag forces are the same as before. The simplification comes in treating the force produced at the kinetochore (always directed poleward) as simple linear force-velocity relationships, with prescribed stall forces and maximum velocities, ignoring the intricacies of polymerisation, depolymerisation and motors. Furthermore, a stochastic process with constant frequencies of attachment and detachment controls the number of kMTs attached to the kinetochore. This minimal mechanical model can reproduce the dynamics of a merotelic fission yeast kinetochore in vivo. It makes the prediction that if three or more of the six kinetochores are merotelic then the spindle would collapse-future observations of live cells will be needed to test this hypothesis. However, the simpli- fications of the Courtheoux et al. model will need development if it is to drive our understanding of the merotelic state. Further work will also certainly be needed to understand the merotelic state in mammalian pre-anaphase and anaphase cells and whether we can be guided by these initial yeast-based models. Again, quantitative analysis of the dynamics of merotelic attached kinetochores is essential to inform the building of further models.

\section{New models, new experiments}

Modelling of chromosome directional instability has demonstrated that there are key (structural) requirements for oscillatory behaviour, specifically a spatially dependent restoration force and a feedback mechanism, while a kinetochore-MT mechanism with a load-dependent variable number of bindings, as in the Hill sleeve, endows the oscillation with realistic constant velocity characteristics. A variety of biological processes can produce the latter, and thus testing out which factors are essential for a given system requires extensive experimentation. Oscillatory behaviour itself is not able to distinguish between mechanisms and asking which processes are essential for oscillations will only provide an entry point for further experiments. We know the minimal requirements for oscillations, but it is very unlikely that the system is minimal, and the key questions are what are the behavioural characteristics of the system and what processes provide that behaviour. The circadian clock field is an excellent exemplar; for decades the minimal requirements for an oscillatory clock have been known whilst only over the last decade has it become clear that behaviour such as tracking both dawn and dusk, and having a period that is tolerant to temperature variation are crucial aspects of the biological clock (Edwards et al. 2010; Gould et al. 2006), characteristics that emerge from a more complex clock network. A detailed analysis of kinetochore oscillatory trajectories and functional dependencies (of amplitude, shape and frequency) can thus determine underlying mechanisms, whilst defining the key behavioural characteristics will require a broader remit for experimentation and modelling, possibly incorporating error correction (merotelic and syntelic attachments), transitions between attachment states, robustness to noise, and 
integration with spindle checkpoint processes. Crucial unresolved questions also remain to be answered: Is kMT dynamic instability the driving force for chromosome directional instability or is kMT (de) polymerization totally controlled through a chemical regulatory network? Which mechanisms and feedbacks are important for what aspects of the behaviour and in which species? Are there functional interactions between proximal chromosomes? How do kinetochores switch between attachment states? And then, the most important question of all-What are the oscillations actually for? We propose that they may reflect the underlying mechanochemistry of the kinetochore and function as a "self-test" on the system to check for the correct balance of forces/ kMT engagement. This could be closely related to experimental work in Drosophila (and an associated mathematical model), which supports the idea that flux-dependent mechanisms could operate to equalise tension across kinetochores within the metaphase plate and that this is important for error-free anaphase (Matos et al. 2009). It will be exciting to see how oscillatory behaviour is coupled to spindle checkpoint activation/inactivation and/or downstream consequences for a chromosome in terms of anaphase fidelity. Other ideas are that the oscillations are crucial to avoid the chromosome arm entanglement that would otherwise be catastrophic for anaphase chromatid separation.

The major limitation to progress in this area is that we are data poor; there is an increasing need for large numbers of quantitative datasets that describe kinetochore trajectories and transitions between attachment states in three dimensions under multiple parameter variations (perturbations) and at varying temporal resolutions. Improvements in the application of fluorescent reporters in human cells, rapidly advancing microscope technology and the development of automated machine vision allows the collection of datasets consisting of hundreds of kinetochore trajectories (Jaqaman et al. 2010). The future should see the development of further live cell assays that can read out the multiple mechanical properties of the kinetochore-k-fibre-spindle pole system and/or report on the dynamics of kinetochore components, including Mad2 (checkpoint activation/inactivation), EB proteins (number of growing kMTs), CENP-H/I (control of kMT dynamics) during kinetochore directional switching. We then need to test models over a range of parameter variations, for example, those invoked by RNAi, somatic gene knockouts and targeted proteolysis methodologies that are workable in human cells (Elbashir et al. 2001; Berdougo et al. 2009; Nishimura et al. 2009). Such advances will open up a new area of data-driven modelling that will necessitate a computationally heavy statistical methodology to integrate models with experimental data. This will lead to an iterative cycle of mathematical modelling and empirical observation, which will be a powerful tool for providing new insight into the mechanisms of chromosome motion control.

Acknowledgments We thank Jonathan Armond, Catarina Samora, Patrick Meraldi, Jonathan Millar and Douglas Drummond for helpful discussions and comments on the manuscript. This work was funded by an EPSRC studentship (E.H.) and a programme grant from Marie Curie Cancer Care (A.M and E.V.).

Open Access This article is distributed under the terms of the Creative Commons Attribution Noncommercial License which permits any noncommercial use, distribution, and reproduction in any medium, provided the original author(s) and source are credited.

\section{References}

Akiyoshi B, Sarangapani KK, Powers AF, Nelson CR, Reichow SL, Arellano-Santoyo H, Gonen T, Ranish JA, Asbury CL, Biggins S (2010) Tension directly stabilizes reconstituted kinetochore-microtubule attachments. Nature 468:576-579

Alushin GM, Ramey VH, Pasqualato S, Ball DA, Grigorieff N, Musacchio A, Nogales E (2010) The Ndc80 kinetochore complex forms oligomeric arrays along microtubules. Nature 467:805-810

Amaro AC, Samora CP, Holtackers R, Wang E, Kingston IJ, Alonso M, Lampson M, McAinsh AD, Meraldi P (2010) Molecular control of kinetochore-microtubule dynamics and chromosome oscillations. Nat Cell Biol 12:319-329

Andrews PD, Ovechkina Y, Morrice N, Wagenbach M, Duncan K, Wordeman L, Swedlow JR (2004) Aurora B regulates MCAK at the mitotic centromere. Dev Cell 6:253-268

Berdougo E, Terret ME, Jallepalli PV (2009) Functional dissection of mitotic regulators through gene targetting in human somatic cells. Methods Mol Biol 545:21-37

Brinkley BR, Ouspenski I, Zinkowski RP (1992) Structure and molecular organization of the centromere-kinetochore complex. Trends Cell Biol 2:15-21

Campas O, Sens P (2006) Chromosome oscillations in mitosis. Phys Rev Lett 97:128102

Cheeseman IM, Chappie JS, Wilson-Kubalek EM, Desai A (2006) The conserved KMN network constitutes the core microtubule-binding site of the kinetochore. Cell 127:983997 
Cimini D, Wan XH, Hirel CB, Salmon ED (2006) Aurora kinase promotes turnover of kinetochore microtubules to reduce chromosome segregation errors. Curr Biol 16:1711-1718

Civelekoglu-Scholey G, Sharp DJ, Mogilner A, Scholey JM (2006) Model of chromosome motility in Drosophila embryos: adaptation of a general mechanism for rapid mitosis. Biophys J 90:3966-3982

Cleveland DW, Mao YH, Sullivan KF (2003) Centromeres and kinetochores: from epigenetics to mitotic checkpoint signaling. Cell 112:407-421

Courtheoux T, Gay G, Gachet Y, Tournier S (2009) Ase1/Prc1dependent spindle elongation corrects merotely during anaphase in fission yeast. J Cell Biol 187:399-412

Cytrynbaum EN, Scholey JM, Mogilner A (2003) A force balance model of early spindle pole separation in Drosophila embryos. Biophys J 84:757-769

Du Y, English CA, Ohi R (2010) The kinesin-8 Kif18A dampens microtubule plus-end dynamics. Curr Biol 20:374-380

Dumont S, Mitchison TJ (2009) Force and length in the mitotic spindle. Curr Biol 19:R749-R761

Edwards KD, Akman OE, Knox K, Lumsden PJ, Thomson AW, Brown PE, Pokhilko A, Kozma-Bognar L, Nagy F, Rand DA, Millar AJ (2010) Quantitative analysis of regulatory flexibility under changing environmental conditions. Mol Syst Biol 6:424

Elbashir SM, Martinez J, Patkaniowska A, Lendeckel W, Tuschl T (2001) Functional anatomy of siRNAs for mediating efficient RNAi in Drosophila melanogaster embryo lysate. EMBO J 20:6877-6888

Gardner MK, Odde DJ (2006) Modeling of chromosome motility during mitosis. Curr Opin Cell Biol 18:639647

Gardner MK, Pearson CG, Sprague BL, Zarzar TR, Bloom K, Salmon ED, Odde DJ (2005) Tension-dependent regulation of microtubule dynamics at kinetochores can explain metaphase congression in yeast. Mol Biol Cell 16:37643775

Gould PD, Locke JCW, Larue C, Southern MM, Davis SJ, Hanano S, Moyle R, Milich R, Putterill J, Millar AJ, Hall A (2006) The molecular basis of temperature compensation in the Arabidopsis circadian clock. Plant Cell 18:1177-1187

Hill TL (1985) Theoretical problems related to the attachment of microtubules to kinetochores. P Natl Acad Sci USA 82:4404-4408

Howard J (2005) Mechanics of motor proteins and the cytoskeleton. Sinauer Associates Inc., U.S

Hughes AF, Swann MM (1948) Anaphase movements in the living cell —a study with phase contrast and polarised light on chick tissue cultures. J Exp Biol 25:45-70

Jaqaman K, King EM, Amaro AC, Winter JR, Dorn JF, Elliott HL, Mchedlishvili N, McClelland SE, Porter IM, Posch M, Toso A, Danuser G, McAinsh AD, Meraldi P, Swedlow JR (2010) Kinetochore alignment within the metaphase plate is regulated by centromere stiffness and microtubule depolymerases. J Cell Biol 188:665-679

Joglekar AP, Hunt AJ (2002) A simple, mechanistic model for directional instability during mitotic chromosome movements. Biophys J 83:42-58
Joglekar AP, Bloom KS, Salmon ED (2010) Mechanisms of force generation by end-on kinetochore-microtubule attachments. Curr Opin Cell Biol 22:57-67

Johnston K, Joglekar A, Hori T, Suzuki A, Fukagawa T, Salmon ED (2010) Vertebrate kinetochore protein architecture: protein copy number. J Cell Biol 189:937-943

Kapoor TM, Compton DA (2002) Searching for the middle ground: mechanisms of chromosome alignment during mitosis. J Cell Biol 157:551-556

Ke K, Cheng J, Hunt AJ (2009) The distribution of polar ejection forces determines the amplitude of chromosome directional instability. Curr Biol 19:807-815

Khodjakov A, Pines J (2010) Centromere tension: a divisive issue. Nat Cell Biol 12:919-923

Khodjakov A, Rieder CL (1996) Kinetochores moving away from their associated pole do not exert a significant pushing force on the chromosome. J Cell Biol 135:315-327

Kops GJ, Saurin AT, Meraldi P (2010) Finding the middle ground: how kinetochores power chromosome congression. Cell Mol Life Sci 67:2145-2161

Lampson MA, Cheeseman IM (2010) Sensing centromere tension: Aurora B and the regulation of kinetochore function. Trends Cell Biol (in press)

Lewis WH (1939) Changes of viscocity and cell activity. Science 89:400

Liu J, Desai A, Onuchic JN, Hwa T (2007) A mechanobiochemical mechanism for monooriented chromosome oscillation in mitosis. P Natl Acad Sci USA 104:16104-16109

Liu J, Desai A, Onuchic JN, Hwa T (2008) An integrated mechanobiochemical feedback mechanism describes chromosome motility from prometaphase to anaphase in mitosis. P Natl Acad Sci USA 105:13752-13757

Loncarek J, Kisurina-Evgenieva O, Vinogradova T, Hergert P, La Terra S, Kapoor TM, Khodjakov A (2007) The centromere geometry essential for keeping mitosis error free is controlled by spindle forces. Nature 450:745-749

Maiato H, DeLuca J, Salmon ED, Earnshaw WC (2004) The dynamic kinetochore-microtubule interface. J Cell Sci 117:5461-5477

Maresca TJ, Salmon ED (2009) Intrakinetochore stretch is associated with changes in kinetochore phosphorylation and spindle assembly checkpoint activity. J Cell Biol 184:373-381

Maskell DP, Hu XW, Singleton MR (2010) Molecular architecture and assembly of the yeast kinetochore MIND complex. J Cell Biol 190:823-834

Matos I, Pereira AJ, Lince-Faria M, Cameron LA, Salmon ED, Maiato H (2009) Synchronizing chromosome segregation by flux-dependent force equalization at kinetochores. J Cell Biol 186:11-26

Mayr MI, Hummer S, Bormann J, Gruner T, Adio S, Woehlke G, Mayer TU (2007) The human kinesin Kif18A is a motile microtubule depolymerase essential for chromosome congression. Curr Biol 17:488-498

McAinsh AD, Tytell JD, Sorger PK (2003) Structure, function, and regulation of budding yeast kinetochores. Annu Rev Cell Dev Biol 19:519-539

Mogilner A, Oster G (2003) Force generation by actin polymerization II: the elastic ratchet and tethered filaments. Biophys J 84:1591-1605

Nicklas RB (1988) The forces that move chromosomes in mitosis. Annu Rev Biophys Biophys Chem 17:431-449 
Nishimura K, Fukagawa T, Takisawa H, Kakimoto T, Kanemaki M (2009) An auxin-based degron system for the rapid depletion of proteins in nonplant cells. Nat Methods 6:917-922

Petrovic A, Pasqualato S, Dube P, Krenn V, Santaguida S, Cittaro D, Monzani S, Massimiliano L, Keller J, Tarricone A, Maiolica A, Stark H, Musacchio A (2010) The MIS12 complex is a protein interaction hub for outer kinetochore assembly. J Cell Biol 190:835-852

Powers AF, Franck AD, Gestaut DR, Cooper J, Gracyzk B, Wei RR, Wordeman L, Davis TN, Asbury CL (2009) The Ndc80 kinetochore complex forms load-bearing attachments to dynamic microtubule tips via biased diffusion. Cell 136:865-875

Santaguida S, Musacchio A (2009) The life and miracles of kinetochores. EMBO J 28:2511-2531

Sharp DJ, Rogers GC, Scholey JM (2000) Microtubule motors in mitosis. Nature 407:41-47

Shtylla B, Keener JP (2010) A mechanomolecular model for the movement of chromosomes during mitosis driven by a minimal kinetochore bicyclic cascade. J Theor Biol 263:455-470

Skibbens RV, Skeen VP, Salmon ED (1993) Directional instability of kinetochore motility during chromosome congression and segregation in mitotic newt lung-cells-a push-pull mechanism. J Cell Biol 122:859-875

Stumpff J, von Dassow G, Wagenbach M, Asbury C, Wordeman L (2008) The kinesin-8 motor Kif18A suppresses kinetochore movements to control mitotic chromosome alignment. Dev Cell 14:252-262

Toso A, Winter JR, Garrod AJ, Amaro AC, Meraldi P, McAinsh AD (2009) Kinetochore-generated pushing forces separate centrosomes during bipolar spindle assembly. J Cell Biol 184:365-372

Uchida KSK, Takagaki K, Kumada K, Hirayama Y, Noda T, Hirota $T$ (2009) Kinetochore stretching inactivates the spindle assembly checkpoint. J Cell Biol 184:383-390

Wan X, O'Quinn RP, Pierce HL, Joglekar AP, Gall WE, DeLuca JG, Carroll CW, Liu ST, Yen TJ, McEwen BF, Stukenberg PT, Desai A, Salmon ED (2009) Protein architecture of the human kinetochore microtubule attachment site. Cell 137:672-684

Wei RR, Schnell JR, Larsen NA, Sorger PK, Chou JJ, Harrison SC (2006) Structure of a central component of the yeast kinetochore: the Spc24p/Spc25p globular domain. Structure 14:1003-1009

Welburn JPI, Grishchuk EL, Backer CB, Wilson-Kubalek EM, Yates JR, Cheeseman IM (2009) The human kinetochore Ska1 complex facilitates microtubule depolymerizationcoupled motility. Dev Cell 16:374-385

Wordeman L, Wagenbach M, von Dassow G (2007) MCAK facilitates chromosome movement by promoting kinetochore microtubule turnover. J Cell Biol 179:869-879

Zhai Y, Kronebusch PJ, Borisy GG (1995) Kinetochore microtubule dynamics and the metaphase-anaphase transition. J Cell Biol 131:721-734 